\title{
Usando el género para criticar al Derecho
}

\author{
Marisol Fernández Revoredo*
}

El género, entendido como una categoría que alude a las construcciones sociales y culturales sobre la base de las diferencias sexuales, ha operado también como una herramienta fundamental para aproximarse críticamente al Derecho. En el ámbito jurídico, esta categoría ha sido formulada por la academia feminista, generando muchas resistencias en nuestro medio. Una manifestación de ello es que en las facultades de Derecho de las universidades latinoamericanas no existen cursos obligatorios en los que se analice el género como un método de análisis jurídico.

A través del presente artículo pretendemos explicar por qué incorporar un enfoque de género en el campo del Derecho es indispensable para comprender la racionalidad de esta disciplina y transformarla. Para ello, presentaremos una síntesis de las diferentes vertientes críticas al Derecho formuladas por el feminismo, para luego cotejar su vigencia en nuestro contexto social y jurídico. ${ }^{1}$ Finalmente, plantearemos algunas ideas acerca de cómo incorporar el enfoque de género y los aportes de la crítica legal feminista en la enseñanza del Derecho.

\section{Las críticas feministas al Derecho}

Una primera cuestión tiene que ver con lo que se entiende por Derecho en estas vertientes críticas. Y es que el Derecho es un concepto amplio, que está integrado — como lo señala Alda Facio, ${ }^{2}$ siguiendo a Margaret Schuler— no

\footnotetext{
Profesora del Departamento Académico de Derecho de la Pontificia Universidad Católica del Perú.

1 Se ha dicho que la crítica feminista al Derecho ha tenido su origen en el ámbito anglosajón, no siendo aplicable a una realidad jurídica como la nuestra.

2 FACIO, Alda. Cuando el género suena cambios trae. Metodología para el análisis de género del fenómeno legal. Primera edición. San José de Costa Rica: ILANUD, 1992, pp. 87-88.
} 
solo por las normas escritas sino también por una dimensión institucional y otra cultural, que se relacionan íntimamente. De tal manera que se encuentran involucradas las instituciones que crean, interpretan y aplican las normas, así como el contenido que le imprimen a las mismas al realizar esas tareas; aunado a ello, están "[...] los contenidos — convertidos en leyes no escritas-, que la gente le da a las leyes y la aplicación de las mismas por medio de las tradiciones, las costumbres, el conocimiento y uso que de ellas hagan». ${ }^{3}$

En la misma línea de Schuler y Facio, Carol Smart define el Derecho de la siguiente manera:

En un nivel, es lo que llega a ser parte de un estatuto como resultado de un proceso político. Obviamente, el derecho establecido está abierto a la interpretación, aunque no a una libre interpretación. Un conjunto de convenciones se aplica a lo que podemos definir como metodología legal. No podemos comprender el derecho sin una valoración crítica de esta metodología. En otro nivel está la práctica del derecho [...]. Me refiero a cómo los abogados y otros actores legales, como la policía usan el derecho - y lo interpretan con menos escrutinio- en la práctica todos los días. Este tipo de derecho se conoce por estar a una gran distancia del derecho en los libros o en el derecho común, pero obviamente no está desvinculado de esto. Pero el derecho es más que la suma de estos elementos. Es también lo que la gente cree que es, en tanto puede guiar sus acciones por él. ${ }^{4}$

Así pues, la crítica feminista al Derecho se dirige a un sistema jurídico en el que las normas, las instituciones y la cultura se nutren mutuamente generando un orden en el que las mujeres terminan perdiendo.

Ya habíamos anunciado que la crítica feminista al Derecho no ha sido monolítica; por el contrario, ha suscitado planteamientos diversos e intensos debates, pero que finalmente convergen en una denuncia por la desvalorización de las mujeres y de lo femenino en el ámbito jurídico. A continuación haremos una muy breve presentación de lo que se postula en cada una de las principales vertientes críticas.

\subsection{El feminismo jurídico liberal}

El problema para las liberales clásicas no ha sido el Derecho en sí mismo sino el trato desigual que las mujeres reciben, de tal modo que el cuestionamiento se ha dirigido a las normas que las relegan al ámbito privado, y que, en general, no las reconocen como sujetos de derecho, como lo son los varones. Bajo esta

Ibíd., p. 89.

4 SMART, Carol. «La teoría feminista y el discurso jurídico». En BIRGIN, Haydée (comp.) El Derecho en el género y el género en el Derecho. Buenos Aires: Editorial Biblos, 2000, pp. 31-32. 
óptica, entonces, las diferencias de género entre varones y mujeres no deben ser relevantes para el ordenamiento jurídico, y por ello, no se justifican diferenciaciones de trato sobre la base del sexo. Este es, precisamente, uno de los aspectos debatidos en relación con las propuestas feministas, pues como se verá más adelante, el feminismo cultural sostiene que más bien las diferencias deben ser celebradas y recogidas por el Derecho.

Como lógica consecuencia de estos planteamientos, el punto central de la agenda del feminismo liberal clásico ha sido la reforma legal, con el propósito de lograr la igualdad de trato entre varones y mujeres. Así pues, la igualdad ante la ley y la prohibición de prácticas discriminatorias por razón de sexo jugarían un papel central, pues serían las garantías de que las mujeres fueran tratadas como sujetos de derecho, igual que los varones.

No nos cabe duda acerca de la importancia que tuvieron, en su momento, los planteamientos antes descritos, teniendo en cuenta el contexto discriminatorio en el que estos surgieron. Sin embargo, como lo explica Jaramillo, sus limitaciones dieron pie al feminismo liberal social, cuyo énfasis está puesto en la desigual distribución de recursos por razón de género y que:

Se concentra entonces en mostrar que si bien las mujeres tienen acceso al empleo, están peor remuneradas que los hombres; tienen jornadas laborales más largas porque no sólo deben atender a su obligaciones frente al trabajo remunerado sino asumir las tareas del cuidado y domésticas; sufren discriminación en el empleo que les impide entrar a ciertas áreas del mercado y ascender [....$^{5}$

Así, en el plano jurídico esta versión liberal propondría una reforma legal hacia la igualdad de oportunidades, con un especial énfasis en el trabajo, siendo una de las herramientas para lograr ello las llamadas acciones afirmativas para compensar las históricas desigualdades entre varones y mujeres.

\subsection{El feminismo jurídico socialista}

La relación del feminismo con el materialismo y el socialismo no ha sido sencilla; por el contrario, existe una historia de desacuerdos. ${ }^{6}$ Bodelón, siguiendo a Sargen, Eisenstein y Mitchell, sostiene que el feminismo socialista encuentra en la retroalimentación entre capitalismo y patriarcado un entramado de condiciones para la explotación de las mujeres. ${ }^{7}$ Así pues, esta vertiente crítica se

5 Jaramillo, Isabel Cristina. «La crítica feminista al Derecho». Estudio preliminar. En Género y teoría del Derecho. Bogota: Siglo del Hombre Editores, 1999.

6 Bodelón, Encarna. La igualdad y el movimiento de mujeres: propuestas y metodología para el estudio del género. Documento de Trabajo n 148. Barcelona: Universitat Autónoma de Barcelona, 1998, p. 4. También en: www.recercat.net

Ibíd., p. 4. 
nutre de la teoría socialista, sumando a ella el reconocimiento de que el género es un factor central de la opresión a las mujeres.

Bajo esta concepción, el trabajo reproductivo de las mujeres en el ámbito familiar — preparar los alimentos, cuidar a los hijos, etc.—, que sirve para reproducir la fuerza de trabajo productivo, es un elemento determinante de la condición subordinada de aquellas.

En este orden de ideas, el feminismo jurídico socialista sostiene que el Derecho, a través de la regulación de diversos temas e instituciones (como las relaciones familiares, el trabajo doméstico y la maternidad, entre otros), es una pieza clave para mantener una división sexual del trabajo, en la cual las actividades de las mujeres quedan devaluadas, alimentando el sistema opresivo.

Sin ánimo de ubicar a Marcela Legarde entre las feministas socialistas, lo explicado por ella en relación con el trabajo de las mujeres nos parece muy importante en esta instancia:

Las mujeres siempre han trabajado. Pero existen dificultades para definir su trabajo, primero porque se le juzga a partir de la división histórica del trabajo, como natural, como característica sexual. Segundo, porque una parte de su trabajo le ocurre y lo hace, en y por mediación de su cuerpo, y no es diferenciada de él como una actividad social creativa. Tercero, porque el resto del trabajo de la mujer, por asociación, es derivado naturalmente del trabajo no concebido como tal. ${ }^{8}$

Así pues, esta concepción del trabajo de la mujer alimenta al Derecho, que a su vez la refuerza mediante sus dimensiones normativa, institucional y cultural.

\subsection{El feminismo jurídico cultural}

La investigación desarrollada por Carol Gilligan y presentada en el libro In a different voice, traducido al castellano bajo el título La moral y la teoría. Psicología del desarrollo femenino, ha tenido una muy importante influencia en los planteamientos críticos al Derecho formulados por el feminismo jurídico cultural.

Gilligan llegó a la conclusión de que varones y mujeres tienden a desarrollar sensibilidades morales de manera distinta, adjudicándoles una ética del cuidado y una ética de la justicia, respectivamente. Explica, en su investigación, que al pedirles a las mujeres que se describan a sí mismas,

[...] todas las mujeres describen una relación, mostrando su identidad en la conexión de madre futura, esposa actual, hija adoptiva o amante pasada. De manera similar, la norma del juicio moral que imbuye su evaluación del

LeGaRde y de los Ríos, Marcela. Los cautiverios de las mujeres: madresposas, monjas, putas, presas y locas. México D. F.: Universidad Autónoma de México, 2003, p. 116. 
Yo es una norma de relación, una ética de alimentación y cría, responsabilidad y cuidado. Midiendo su fuerza en las actividades del apego, dar, ayudar, ser bondadosa, no causar daño, estas mujeres, que han triunfado y se han realizado, no mencionan su distinción académica y profesional al describirse a sí mismas. ${ }^{9}$

En esa misma línea, sostiene Gilligan que las mujeres que formaron parte de la investigación antes citada «[...] consideran que la moral brota de la experiencia de la conexión y es concebida como problema de inclusión, y no de sopesar derechos». ${ }^{10}$

Por otra parte, la identidad de los hombres está marcada por la realización individual. En vez del apego, es la separación lo que define el $Y o$ varonil; como correlato, tienen un razonamiento moral distinto. En suma, Gilligan resume esta dicotomía de las experiencias morales de la siguiente manera:

[...] estas diversas perspectivas se reflejan en dos diferentes ideologías morales, ya que la separación queda justificada por una ética de derechos, mientras que el apego es apoyado por una ética del cuidado y la atención. La moral de los derechos se basa en la igualdad y se centra en la comprensión de la imparcialidad, mientras que la ética de la responsabilidad se basa en el concepto de igualdad y reconocimiento de las diferencias de necesidad. Mientras que la ética de los derechos es una manifestación de igual respeto, que equilibra los derechos de los otros y del Yo, la ética de la responsabilidad se basa en un entendimiento que hace surgir la compasión y el cuidado. ${ }^{11}$

Decíamos pues que el feminismo jurídico cultural ${ }^{12}$ se nutre de los planteamientos de Gilligan y denuncia que:

Los principios de imparcialidad, neutralidad y objetividad son considerados principios masculinos, desarrollados con el objetivo de ocultar la parcialidad de la ley, su preferencia por los varones y su visión del mundo. El contenido de la ley, la forma cómo opera, la cultura de la subjetividad y la autonomía, el modelo adversarial y la organización jerárquica del sistema son aspectos que reflejan la masculinidad del Derecho y su carácter de institución patriarcal. Creado por

\footnotetext{
Gilligan, Carol. La moral y la teoría. Psicología del desarrollo femenino. México D. F.: Fondo de Cultura Económica, 1994, p. 258.

$10 \quad$ lbíd., p. 259.

11 Ibíd., pp. 265-266.

12 West señala que desde la psicología del desarrollo se plantea pero no se explica el por qué las mujeres crían y sustentan a sus hijos y los hombres no. Sostiene, sin embargo, que otras feministas culturales han respondido a esa interrogante de manera diversa, pero sus explicaciones convergen, implícita sino explícitamente, en una respuesta material, o combinada entre lo material y lo cultural, y no solamente cultural.
} 
varones a su imagen y semejanza, el derecho asegura su posición de dominio. La neutralidad y la objetividad sirven para oscurecer su sesgo masculino. ${ }^{13}$

Una cuestión que hay que resaltar es que, subyace a esta crítica, una especial valoración de las diferencias de género entre varones y mujeres. La diferente socialización por razón de género, junto con factores como la maternidad, determinan para las feministas culturales diferentes visiones y formas de actuar. Si el Derecho las tomara en cuenta, variaría el contenido de las leyes, su aplicación, así como la manera de resolver los conflictos.

Si nos trasladamos al campo de las estrategias para cambiar este Derecho masculino, vamos a encontrar posiciones divergentes que van desde incorporar la perspectiva de las mujeres al sistema jurídico, hasta eliminar al patriarcado como condición para un nuevo Derecho que incluya las experiencias de varones y mujeres. ${ }^{14}$

\subsection{El feminismo jurídico radical}

Catherine MacKinnon es una de las más importantes exponentes de la crítica feminista radical. Ella sostiene:

La fisiología de los hombres define la mayoría de los deportes, sus necesidades definen la cobertura de los seguros médicos y de automóviles, sus biografías sociales definen las expectativas en los lugares de trabajo y los patrones exitosos de carrera, sus perspectivas y preocupaciones definen la calidad de la producción académica, sus experiencias y obsesiones definen el mérito, su objetivización de la vida define el arte, su servicio militar define la ciudadanía, su presencia define a la familia, su incapacidad para llevarse bien con los demás — sus guerras y gobierno- define la historia, su imagen define a dios, y sus genitales definen el sexo. ${ }^{15}$

Esta descripción, pues, alude a una sociedad articulada bajo un sistema de dominación patriarcal, lo que constituye la gran denuncia que hace el feminismo radical.

MacKinnon ha puesto el énfasis en la sexualidad para explicar el origen de la dominación masculina, señalando que el Derecho juega un papel central al reproducir la sexualidad desde la perspectiva de los varones, garantizando el

13 Kohen, Beatriz. «El feminismo jurídico en los países anglosajones: el debate actual». En Birgin, Haydée. El Derecho en el género y el género en el Derecho. Buenos Aires: Editorial Biblos, 2000, p. 86.

14 Ibíd., p. 87.

15 MacKInNON, Catherine. «Diferencia y dominio: sobre la discriminación sexual» (1984). En Navarro, Marysa y Stimpson, Catherine (compiladoras). Sexualidad, género y roles sexuales. Buenos Aires: Fondo de Cultura Económica, 1999, pp. 84-85. 
control sobre el cuerpo de las mujeres. Ello explica que las feministas radicales hayan centrado su atención en temas como la pornografía, la prostitución, la violación y el acoso sexual. Así pues, la intervención en el campo del Derecho no pasa por una reforma legal sino por refundar el sistema jurídico.

Robin West hace una especie de análisis comparativo de las concepciones que subyacen al feminismo cultural y radical, señalando sobre este último lo siguiente:

$\mathrm{Al}$ feminismo radical, entonces, lo une una descripción de la subjetividad del estado material de conexión. Según esa descripción, las mujeres temen la intrusión y la invasión y anhelan una identidad independiente, individualizada y separada. Aunque es posible que, en efecto, las mujeres valoren oficialmente la intimidad, lo que ellas ansían de manera no oficial es la autonomía y la integridad físicas, así como el celibato: en una palabra la exclusividad física. En el campo moral, las mujeres valoramos oficialmente una reflexión moral contextual, relacional, del cuidado, pero secretamente deseamos que todo el mundo se vaya al diablo para que podamos continuar con nuestros propios proyectos: aborrecemos la intrusión que la intimidad acarrea. ${ }^{16}$

\section{Las críticas feministas y el ordenamiento jurídico peruano}

No nos cabe duda de que un análisis del ordenamiento jurídico peruano, utilizando el género y otros conceptos propuestos por las corrientes críticas feministas antes explicadas, nos permitirían descubrir en el Derecho peruano aspectos y dimensiones que no lograríamos ver con un análisis carente de esas categorías. Tomando las palabras de Alda Facio, «Con los lentes de género se ve otro Derecho».

De tal manera que la tarea pendiente, en el estudio de nuestro ordenamiento jurídico, es la de dar cuenta del enfoque y los métodos del feminismo jurídico para poder completar su caracterización. Ello, además, servirá para una retroalimentación de la propia teoría feminista.

A nuestro modo de ver los puntos más críticos del Derecho y las instituciones con la que debemos iniciar esta tarea, son las siguientes:

1. El derecho constitucional

2. El derecho de familia

3. El derecho laboral

4. El derecho penal

16 WEST, Robin. Género y teoría del Derecho. Bogotá: Siglo del Hombre Editores, 1999, p. 122. 
En el marco del derecho constitucional debe evaluarse el impacto, en el Perú, de la constitucionalización del principio de igualdad y no discriminación para las mujeres, así como las medidas de acción afirmativa. Si el problema del Derecho fuera el trato desigual para las mujeres, así como la falta de oportunidades, la aplicación de tales principios debió conducir a reformas legales, que a su vez habrían eliminado el sexismo del Derecho. La Constitución de 1979 marcó un hito muy importante al crear las condiciones para modificar normas y prácticas discriminatorias contra las mujeres; la interrogante que hay que resolver es si los cambios normativos trajeron consigo le erradicación del sexismo en el fenómeno legal. Sobre el particular, creemos que la Constitución de 1979 fue la semilla que germinó la preocupación por la inserción de las mujeres en el ámbito público, particularmente en lo que atañe a su participación política, ${ }^{17}$ por la formulación de planes y políticas encaminados a la igualdad de oportunidades, y por un cuestionamiento al modelo familiar de potestad marital plasmado en nuestro ordenamiento familiar. Sin embargo, si en el marco del nuevo orden nacido a partir de las reformas legales promovidas a la luz de la mencionada Constitución, nos hacemos la pregunta por las mujeres, la respuesta ineludiblemente nos devuelve, aún, a una realidad con discriminación y violencia de género.

Hay quienes sostienen, a partir del desarrollo normativo de la igualdad en diferentes terrenos de nuestro ordenamiento, que hemos logrado la neutralidad y que, en consecuencia, el problema habría que centrarlo en la aplicación por parte de los operadores, quienes serían los que introducirían el sesgo discriminatorio. Creemos pendiente la tarea de discutir esta premisa, a la luz de la formulación de ciertos derechos e instituciones, que revelarían haber sido concebidos por y para un ser masculino.

Pero el estudio del derecho constitucional con enfoque de género, nos debe llevar también al análisis de las normas y prácticas que determinan la organización del poder político. Marcela Rodríguez nos explica que: «Considerar al género como una categoría analítica en la teoría política y, particularmente, en la teoría del Estado, permite reconstruir, reconceptualizar y ampliar el ámbito y el alcance de la política, sus instituciones y prácticas, conceptos y paradigmas»..$^{18}$

En lo que respecta al derecho de familia peruano, se tiende a señalar que el modelo familiar de potestad marital ya fue superado y que hoy las mujeres están en igualdad de condiciones al interior de la familia. La superación del

Una manifestación de ello es la ley de cuotas de participación política que ha permitido un significativo aumento de mujeres en espacios de gobierno y de formulación de normas.

18 Rodríguez, Marcela. «Diseño institucional, presidencialismo y parlamentarismo, hay alguna diferencia... para las mujeres». En FAcIO, Alda y FrIEs, Lorena (editoras). Género y Derecho. Santiago: LOM Ediciones, 1999, p. 289. 
referido modelo familiar se habría debido a la constitucionalización de la igualdad y de la no discriminación en el año 1979. Un clásico en el derecho de familia peruano ha sido la obra del doctor Héctor Cornejo Chavez, ${ }^{19}$ que además es la fuente a la cual tradicionalmente se ha acudido para el estudio de ese derecho. En relación con los cambios en el ordenamiento familiar, Cornejo afirma que «[...] el nuevo Código ha debido implementar la norma constitucional que proclama la igualdad del varón y la mujer ante la ley y modificar, en consecuencia, la regulación arriba sintetizada $»^{20}$ - la regulación a la que se refiere es la del Código Civil de 1936 que recogía el modelo de potestad marital—.

Así pues, la recepción de la igualdad entre varón y mujer por parte del ordenamiento jurídico familiar, parece dejar tranquilos a los estudiosos y operadores del Derecho. Sin embargo, utilizando el género en el análisis, saltarían a la vista varias cuestiones. Una de las más relevantes es la concepción, recogida por el Derecho, del trabajo de la mujer. El propio Cornejo, luego de reconocer que la igualdad ha sido recogida por el ordenamiento familiar, señala que: «El trabajo productivo es en el ser humano herramienta de dignificación, no un fin en sí mismo. El hombre puede valer más o menos que lo que produce. El trabajo productivo es o debe ser instrumento de liberación. Pero no es el único trabajo digno del ser humano [...] la madre puede no producir bienes tangibles o prestar servicios de valor cuantificable, pero su empeño es también liberadons. ${ }^{21}$ Más adelante sostiene "La solución no es sacar a la madre del hogar. Es elevarla dentro de él. La solución no es que la mujer deba trabajar porque el marido no gana lo suficiente. Es elevar el nivel del salario de éste en función de sus cargas familiares ${ }^{22}$ (las cursivas en ambas citas son nuestras).

Nótese cómo se concibe que el trabajo de la mujer no es cuantificable, y además, que aquella debería mantenerse en ese rol. Esta concepción está impregnada en nuestra legislación, pero invisibilizada por un ropaje que aparenta la superación de la división sexual del trabajo en nuestro ordenamiento. ${ }^{23} \mathrm{~A}$

19 Fue miembro de la Comisión Reformadora que preparó el Código Civil de 1984 y autor de la ponencia del Libro de Familia de ese mismo código.

20 Cornejo Chavez, Hector. Derecho familiar peruano. Tomo I. 8. a ed.. Lima: Librería Studium S. A., 1991, p. 263.

21 Ibíd., p. 265.

22 Ibíd.

23 A nuestro juicio, hay dos disposiciones en el Código Civil que son reveladoras de la concepción descrita sobre el rol de la mujer en la familia, y en particular, de cómo se ha concebido su trabajo. Se trata del artículo 291 que establece que «Si uno de los cónyuges se dedica exclusivamente al trabajo del hogar y al cuidado de los hijos, la obligación de sostener a la familia recae sobre el otro, sin perjuicio de la ayuda y colaboración que ambos cónyuges se deben en uno y otro campo" Asimismo, lo previsto en el artículo 293 que señala que: «Cada cónyuge puede ejercer cualquier profesión o industria permitidos por la ley, así como efectuar cualquier trabajo fuera del hogar, con el asentimiento expreso o tácito del otro. Si éste lo negare, el juez puede autorizarlo, si lo justifica el interés de la familia». 
nuestro entender, esta visión de la mujer y de su trabajo en el ámbito doméstico responde a la identificación mujer-naturaleza que Legarde explica como sigue:

La homologación de las actividades de la mujer con los hechos procreadores que le ocurren, como hechos naturales, conlleva a definir la esencia de cualquier trabajo femenino como sexual, biológica (no humana). Por contagio se anula todo el trabajo femenino. Así el trabajo de la mujer se constituye en mucho más que una característica sexual: es sexualidad femenina, queda subsumido y negado en la feminidad-naturaleza. ${ }^{24}$

A nuestro juicio, la dicotomía trabajo productivo - trabajo reproductivo, recogida y consolidada por el Derecho, constituye un tema central en la caracterización de la condición de las mujeres. De esta división sexual del trabajo - que opera como eje organizador de la familia — depende en gran parte la ideología del mercado, ${ }^{25} \mathrm{de}$ tal modo que nos resultan indispensables para el análisis, las categorías usadas por el feminismo jurídico socialista.

Un nuevo orden jurídico familiar, que recoja en las normas, instituciones y cultura, un modelo de responsabilidades familiares compartidas entre varones y mujeres, rompería con uno de los factores más importantes de la dominación de las mujeres. Ello nos lleva a plantear que el derecho familiar y laboral, aun cuando nos remiten a dinámicas y relaciones muy distintas, deben ser percibidos como un entramado que hoy genera opresión, pero que podrían ser los espacios que contribuyan al cambio.

En la enseñanza del derecho laboral, se suele considerar que lo que compete a la dimensión de género está contenido en la legislación protectora de la maternidad y en la igualdad y no discriminación en el ámbito laboral. Ello, desde las perspectivas críticas que estamos trabajando, resulta una visión muy limitada, pues da por sentada la neutralidad en la formulación de los derechos y que dicha neutralidad es buena para las mujeres trabajadoras. En efecto, derechos como la jornada de trabajo y la regulación de las faltas justificadas al centro laboral, entre otros, han sido creados bajo la concepción de que el trabajador es un varón, lo cual genera que las mujeres trabajadoras que tienen hijos vean afectadas desproporcionadamente sus vidas, en comparación con los trabajadores que son padres de familia.

Ambas disposiciones las hemos comentado en: Fernández, Marisol. «¿La neutralidad de las normas de derecho familiar garantizan la no discriminación por razón de sexo-género? A propósito de los efectos personales del matrimonio». En El Derecho, año XCl, $\mathrm{n}^{\circ} 306$, Colegio de Abogados de Arequipa, diciembre 2005, pp. 201-207.

24 LEGARDE, Marcela, ob. cit. p. 116.

25 OLSEN, Frances. «El sexo del Derecho». En RuIz, Alicia (comp.). Identidad femenina y discurso jurídico. Buenos Aires: Editorial Biblos, 2000, p. 41. 
En términos generales, el derecho laboral que tradicionalmente se enseña, no contempla entre sus contenidos categorías de análisis que permitan cuestionar el impacto de género de las relaciones laborales en el marco de un sistema capitalista, con lo cual se contribuye a perpetuar dinámicas en el mercado que excluyen a las mujeres. El reciente régimen establecido para las trabajadoras del hogar es un ejemplo de la connotación que en el mundo del trabajo tiene el que realizan mayoritariamente las mujeres.

Si en la aproximación al derecho al trabajo nos formuláramos la pregunta por el impacto de género, o específicamente por la mujer, llegaríamos probablemente a diagnósticos distintos a los que contamos el día de hoy.

Por último, para el feminismo jurídico el derecho penal ha significado un orden simbólico que ha contribuido a controlar la sexualidad de las mujeres. Este derecho nos trae un discurso sobre la conducta de las mujeres, contribuyendo así a la construcción del sujeto mujer. Una revisión de la legislación penal y de los discursos construidos por los operadores alrededor de esta es un buen comienzo para una mirada crítica.

\section{El género en la enseñanza del Derecho}

Decíamos al iniciar este artículo que el género ha sido un gran ausente en la malla obligatoria de las currículas de las facultades de Derecho en América Latina. Su presencia ha quedado circunscrita a unas pocas universidades y básicamente como parte de los cursos electivos, con lo cual el impacto que han tenido ha sido mínimo. Consideramos que ello obedece principalmente a dos factores: el primero es que la enseñanza del Derecho se ha centrado en las normas jurídicas. Como lo sostiene Félix Morales,

[...] en este modelo el objetivo es lograr que los alumnos conozcan y comprendan las normas de un determinado sistema jurídico; es decir, dicha enseñanza supone el estudio de las normas, particularmente las leyes. Los estudiantes deben conocer y saber explicar el contenido del ordenamiento jurídico; para ello, el principal material de enseñanza serán las propias normas [....$^{26}$

Esta concepción del Derecho y de su enseñanza resulta siendo un escenario nada propicio para que el género sea utilizado, pues este más bien se ajusta a una visión del Derecho como un entramado de normas, instituciones, valores y cultura, que se nutren mutuamente, recogiendo y retroalimentando un sistema de sexo-género. Ahora bien, este planteamiento nos podría llevar a concluir que como consecuencia de adoptar en el medio académico una concepción del

26 Morales Luna, Félix. «Cambios en Derecho, cambios en su enseñanza». En Derecho PUC, $n^{\circ}$ 58, Lima: Fondo Editorial de la Pontificia Universidad Católica del Perú, 2006, p. 437. 
Derecho y de su enseñanza, como práctica social, el género formaría parte ineludible de las categorías de análisis de las normas, las instituciones y las prácticas. Ello parece ser muy optimista, pues el segundo factor que hay que considerar, por el cual el género no ha sido incorporado en la enseñanza del Derecho, es que la teoría feminista, por la ideología y propuestas políticas que entraña, genera una serie de resistencias en el medio académico, que se repiten para enfrentar, también, al feminismo jurídico.

No incluir al género como teoría y método en la enseñanza-aprendizaje del Derecho produce una serie de consecuencias que deberían ser tomadas en cuenta al diseñar una currícula, entre las que destacan el que se pierda de vista que «[...] el derecho participa en la configuración del estereotipo mujer, y es a partir de ese estereotipo como las reglas jurídicas reconocen o niegan derechos a las mujeres de carne y hueso», ${ }^{27}$ así como que no se comprenda a cabalidad que el género recoge cultura, la transforma en un discurso jurídico y contribuye así a perpetuar una sociedad sexista y discriminatoria para las mujeres. Ello, en otras palabras, supone no formar a los operadores en la idea de que el Derecho puede consistir en una práctica social que puede contribuir a generar importantes transformaciones.

Quienes suscribimos la exigencia - por las razones antes expuestas - de que el género debe jugar un rol protagónico en la formación de los operadores del Derecho, debemos pensar en cómo lograrlo. En nuestra opinión hay dos estrategias. La primera supone que el cambio se geste de arriba hacia abajo. Ello supone que quienes se encargan de la gestión de nuestras facultades replanteen su visión, misión y objetivos institucionales, y que luego esto sirva de base para reelaborar una estructura curricular que lleve a formar a operadores cuestionadores de un orden sexista y conscientes del bagaje cultural que pueden imprimir al aplicar las normas, así como al crearlas.

Ello nos parece difícil de realizar, sobre todo cuando los órganos de gobierno en nuestras facultades de Derecho no han tenido en cuenta el género para su composición. Así pues, la estrategia más viable nos parece aquella en la que el cambio se gesta de abajo hacia arriba, es decir, cuando las profesoras y los profesores, en el ejercicio de su libertad de cátedra, van incorporando a través de módulos específicos en los cursos a su cargo o transversalmente a todo el contenido temático de estos, el enfoque de género. Si bien esta constituye la alternativa más viable, no está exenta de una serie de dificultades, como el vencer las resistencias que impiden lograr un acercamiento entre la perspectiva de género y la teoría feminista. ${ }^{28}$

27 Ruız, Alicia. «De las mujeres y el Derecho». En Ruız, Alicia (comp.). Identidad femenina y discurso jurídico. Buenos Aires: Editorial Biblos, 2000, p. 10.

28 Es importante señalar que se están gestando algunas iniciativas entre las académicas feministas con el objetivo de lograr que en las facultades de Derecho se vaya introduciendo el 
Pero incorporar la perspectiva de género en la enseñanza-aprendizaje del Derecho, no solo supone modificar contenidos temáticos sino introducir nuevos métodos. De un lado, dicha perspectiva supone ver con otros ojos al fenómeno legal, redimensionando el papel de las instituciones y la cultura, al lado de las normas, así como dar cuenta de las metodologías que ha propuesto el feminismo jurídico. ${ }^{29}$

Aunado a lo anterior, en lo que respecta a lo que podríamos llamar el manejo del aula, la metodología de aprendizaje-enseñanza debe ser modificada. Ello supone tomar en cuenta que las diferencias de género están presentes en las aulas, por lo que deben ser tomadas en consideración al definir las relaciones alumnas y alumnos con sus profesoras y profesores. Así, las metodologías activas - centradas en las y los estudiantes, donde se involucra tanto el trabajo individual como el cooperativo y donde se valoran las diferentes formas de aproximarse a un problema jurídico- son la que deberían asumirse en la tarea de enseñar Derecho con perspectiva de género.

\section{Conclusión}

Las críticas feministas al Derecho nos aportan una serie de conceptos, categorías y herramientas que resultan indispensables en una teoría jurídica que se nos presenta como abstracta y neutral, pero que termina siendo una construcción en las que las mujeres no estamos implicadas. El gran reto es que los cambios en la enseñanza del Derecho supongan la incorporación de estos conceptos, para no seguir reproduciendo una realidad excluyente e injusta.

género como un eje transversal. La Red Alas, por ejemplo, llevó a cabo dos talleres con profesoras/es de Derecho en la Universidad San Carlos de Guatemala y en el ITAM en México, en agosto del presente año. Esta experiencia se estaría repitiendo en el Departamento Académico de Derecho de la PUCP.

29 Sobre el particular se puede consultar, entre otros: FACıO, Alda. «Metodología para el análisis de género del fenómeno legal». En FACIO, Alda y FRIES, Lorena (editoras). Género y Derecho. Santiago: LOM Ediciones, 2000, pp. 99-136; y BARTLETT, Katharine. «Feminist Legal Method». En Harvard Law Review 103, pp. 829-887. 